\title{
DYNAMICS OF DAMAGE IN TWO-DIMENSIONAL STRUCTURES WITH WAITING LINKS
}

\author{
Andrej Cherkaev \\ and Liya Zhornitskaya
}

Keywords: Dynamics of damage, Failure, Structures.

\begin{abstract}
The paper deals with simulation of damage spread in special structures with "waiting links." These structures are stable against dynamic impacts due to their morphology. They are able to transform "partial damage" through a large region, thereby dissipating the energy of the impact. We simulate various structures with waiting links and compare their characteristics with conventional designs.
\end{abstract}

\section{INTRODUCTION}

This paper describes protective structures that exhibit an unusually high dissipation when they are subject to a concentrated (ballistic) impact. Such a structure is defined as an assembly (network) of rods connected in knots and submerged into a viscous substance.

During the impact (hit), the kinetic energy of the projectile must be absorbed in the structure; the structure fails if it is unable to absorb the energy. While theoretically a material can absorb energy until it melts, real structures are destroyed by a tiny fraction of this energy due to material instabilities and an uneven distribution of the stresses throughout the structure.

We want to find a structure that absorbs maximal kinetic energy of the projectile without rupturing or breaking. The increase of the stability is achieved due to special structural elements, "waiting links," see [1]. These elements contain parts that are initially inactive and start to resist when the strain is large enough; they lead to large but stable pseudoplastic strains. Structures with "waiting links" distribute the strain over a large area, in contrast to conventional unstructured solids in which the strain is concentrated near the zone of an impact. 


\section{EQUATIONS AND ALGORITHMS}

\subsection{WAITING ELEMENTS}

Brittle-elastic bar. Consider a stretched rod from a homogeneous elastic-brittle material. If loaded, this material behaves as a linear elastic, unless the length $z$ reaches a critical value $z_{f}$, and fails (becomes damaged) after this. The critical value $z_{f}$ is proportional to the length $L$ of the rod at equilibrium

$$
z_{f}=L\left(1+\epsilon_{f}\right)
$$

where the critical strain $\epsilon_{f}$ is a material constant. If the rod is monotonically elongated, the force $F_{\text {monotone }}$ depends on its length $z$ as

$$
F_{\text {monotone }}(z)= \begin{cases}k s(z / L-1) & \text { if } z<z_{f} \\ 0 & \text { if } z \geq z_{f}\end{cases}
$$

where $k$ is an elastic modulus and $s$ is the cross-section of the rod.

Damage parameter. Generally, the elongation of an element is nonmonotone. In order to model the state of damageable rods, we add a principle: Once damaged, the rod stays damaged forever. Namely, we assume that the force $F$ in a rod depends on its length $z$ and on the damage parameter $c$ :

$$
F(z, c)=k s(1-c)(z / L-1)
$$

where $K$ is the elastic modulus. Damage parameter $c$ is equal to zero if the rod is not damaged and is equal to one if the rod is destroyed. Development of the damage is described as the increase of the damage parameter $c(z, t)$ from zero to one. The damage parameter can only increase in time; the increase occurs only when the length of the rod exceeds the critical value. The damage parameter stops increasing when the element is completely damaged. This formalism is similar to the description of damage suggested in [2] for brittle-elastic continuum.

We suggest describing the increase of the damage parameter by the simple differential equation

$$
\frac{d c(z, t)}{d t}=\left\{\begin{array}{ll}
v_{d} & \text { if } \quad z \geq z_{f} \text { and } c<1 \\
0 & \text { otherwise }
\end{array}, \quad c(0)=0\right.
$$

where $z_{f}$ is the maximal elongation that the element can sustain without being damaged, and $v_{d}$ is the speed of damage. The speed $v_{d}$ can be chosen as large as needed. 
Remark 1 The dependence 1.2 corresponds to the discontinuous damage parameter $c_{H}$ that is equal to zero if the element is undamaged and to one if it is damaged:

$$
c_{H}(z, t)=\lim _{v_{d} \rightarrow \infty} c(z, t) .
$$

Consideration of continuously varying damage parameter instead of a discontinuous one increases stability of the computational scheme.

One can argue about the behavior of the rod with an intermediate value of the damage parameter. We do not think that these states need a special justification: they simply express the fact that stiffness rapidly deteriorates to zero when the sample is over-strained.

Waiting links. Let us introduce special structural elements waiting links that significantly increase the resistivity of the structure due to their morphology. These elements and their quasistatic behavior are described in [1]. The link is an assembly of two elastic-brittle rods, lengths $L$ and $\Delta(\Delta>L)$ joined by their ends. The longer bar is initially slightly curved to fit. When an increasing external elongation stretches the link, only the shortest rod resists in the beginning. If the elongation exceeds the critical value, this rod breaks and the next (longer) rod then assumes the load replacing the broken one (see Figure 1,left).
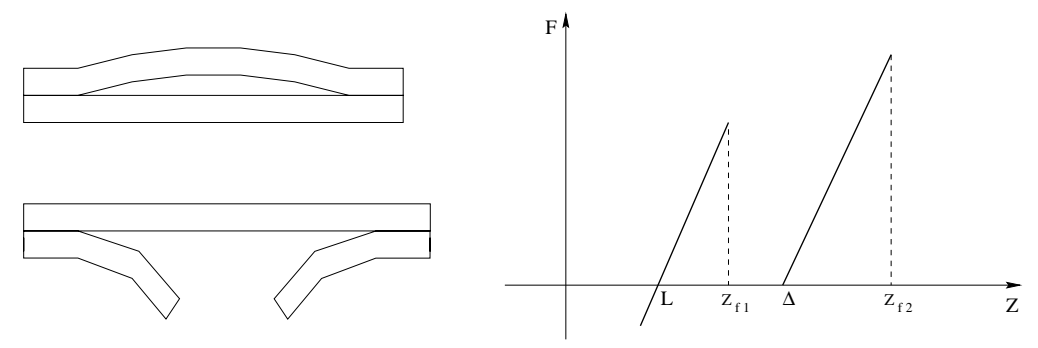

Figure 1 Left above: The waiting link in the initial state. Left below: The waiting link after the first rod is broken. Right: The force-versus-length dependence for a monotone elongation

Assume that a unit amount of material is used for both rods. This amount is divided between the shorter and longer rod: The amount $\alpha$ is used for the shorter (first) rod and the amount $1-\alpha$ is used for the longer (second) one. The cross-sections $s_{1}$ and $s_{2}$ of rods are:

$$
s_{1}(\alpha)=\frac{\alpha}{L} \quad \text { and } s_{2}(\alpha)=\frac{1-\alpha}{\Delta},
$$


The force-versus-elongation dependence in the shorter rod is:

$$
F_{1}(z)=k s_{1}(\alpha)\left(\frac{z}{L}-1\right)\left(1-c_{1}\right)
$$

where $c_{1}=c_{1}(z, t)$ is the damage parameter for this rod; it satisfies the equation similar to (1.4)

$$
\frac{d c_{1}(z, t)}{d t}=\left\{\begin{array}{lll}
v_{d} & \text { if } \quad z \geq z_{f_{1}} \text { and } c_{1}(z, t)<1 \\
0 & \text { otherwise }
\end{array} \quad c_{1}(z, 0)=0\right.
$$

where $z_{f_{1}}=L\left(1+\epsilon_{f}\right)$.

The longer rod starts to resist when the elongation $z$ is large enough to straighten this rod. After the rod is straight, the force-versus-elongation dependence is similar to that for the shorter rod:

$$
F_{2}(z)= \begin{cases}k s_{2}(\alpha)\left(\frac{z}{\Delta}-1\right)\left(1-c_{2}\right), & \text { if } z \geq \Delta \\ 0, & \text { if } z<\Delta\end{cases}
$$

Here $F_{2}$ is the resistance force and $c_{2}=c_{2}(z, t)$ is the damage parameter for the second rod:

$$
\frac{d c_{2}(z, t)}{d t}=\left\{\begin{array}{lll}
v_{d} & \text { if } \quad z \geq z_{f_{2}} \text { and } c_{2}(z, t)<1 \\
0 & \text { otherwise } & c_{2}(z, 0)=0
\end{array}\right.
$$

These equations are similar to (1.6), (1.7), where the cross-section $s_{1}(\alpha)$ is replaced by $s_{2}(\alpha)$ and the critical elongation $z_{f_{1}}$ by $z_{f_{2}}=\Delta\left(1+\epsilon_{f}\right)$. The difference between the two rods is that the longer (slack) rod starts to resist only when the elongation is large enough.

The total resistance force $F(z)$ in the waiting link is the sum of $F_{1}(z)$ and $F_{2}(z)$ :

$$
F(z)=F_{1}(z)+F_{2}(z) .
$$

The graph of this force-versus-elongation dependence for the monotone external elongation is shown on Figure 1 where the damage parameters jump from zero to one at the critical point $z_{f_{1}}$.

One observes that the constitutive relation is nonmonotone. Therefore one should expect that the dynamics of an assembly of such elements is characterized by abrupt motions and waves (similar systems but without damage parameter were investigated in $[5,6])$.

\subsection{STRUCTURES}

Now we describe the model structure: a two-dimensional assembly of the waiting links. Consider a unilateral triangular grid: each inner point 
has six equally distant neighbors. The distance between neighboring knots is equal to $L$. The knots in the boundary (including corners) have lower number of neighbors. The waiting links connect the neighboring knots. Initially, the system is in equilibrium and does not have any inner stress.

If the strain is small everywhere and each link is strained less than the critical value $z_{f}$, the system is linear and it models a linear elastic material. After the first rod in a link breaks and is replaced with a longer one, the net experiences an "irreversible phase transition."

After each break, the network changes its elastic properties and its equilibrium position. The dynamics of the damage and failure of the net can be viewed as a series of these transitions. In contrast with the conventional structure, the waiting links become stronger after first break. Another advantage is that the additional slackness that is added after the break helps to spread the damage across the structure.

Remark 2 In our model, an assembly of a finite number of heavy concentrated masses and weightless damageable springs is studied instead of a continuous structure. The model does not require any a priori assumptions on the thermodynamics or mechanical properties of the modeled media. On the contrary, the properties of equivalent media can be derived by homogenization of the finite model, as the elasticity equation can be derived from the mass-spring model (see, for example, [3]). From this viewpoint, the model is similar to the model by Slepyan [4] of crack dynamics.

Dynamics and Viscosity. It is assumed that the inertial masses $m_{i}$ are concentrated in the knots, joined by the inertialess waiting links (nonlinear springs), therefore the dynamics of the structure is described by ordinary differential equations of motion of the knots. We assume that the links are elastic-brittle, as described above.

Additionally, we assume that the space between the knots is filled with a viscous substance with the dissipation coefficient $\gamma$. Without the viscosity, the system never reaches a steady state. On the other hand, even a slow external excitation leads to intensive waves in the system caused by breaking of the links. These waves, reflected from the boundaries, cause intensive vibration of the structure and the viscosity eventually absorbs the energy.

The motion of the $i$ th knot satisfies the equation

$$
m_{i} \ddot{\mathbf{z}_{\mathbf{i}}}+\gamma \dot{\mathbf{z}_{\mathbf{i}}}=\sum_{j \in N(i)} \frac{F_{i j}\left(\left|\mathbf{z}_{\mathbf{i}}-\mathbf{z}_{\mathbf{j}}\right|\right)}{\left|\mathbf{z}_{\mathbf{i}}-\mathbf{z}_{\mathbf{j}}\right|}\left(\mathbf{z}_{\mathbf{i}}-\mathbf{z}_{\mathbf{j}}\right)
$$


where $\mathbf{z}_{\mathbf{i}}$ is the vector of coordinates of the $i$ th knot, $|$.$| is length of the$ vector, $N(i)$ is the set of knots neighboring the knot $i$ and $m_{i}$ is the mass of the $i$ th knot. The force $F_{i j}$ in the $i j$ th link depends on the damage parameters $c_{i j, 1}$ and $c_{i j, 2}$ as in (1.10). The set of neighbor knots depends on the geometric configuration.

Remark 3 In this model, the masses are permitted to travel as far as the elastic links permit. In particular, when these links are completely broken, the concentrated mass can move "between" other masses without interaction with them. Below in Section 3.1, we discuss a special model for a projectile that is "large enough" so that it does not slip through the rows of linked masses.

Waves of damage. The speed of waves in a structure is of the order of the speed of sound in the material which the structure is made of (approximately 2,000 m/sec for steel). In our numerical experiments, we assume that the speed of the impact is much smaller (recall that the speed of sound in the air is $336 \mathrm{~m} / \mathrm{sec}$ ). A slow-moving projectile does not excite intensive waves in stable structures, but it does excite mighty waves of damage in waiting structures. The reason is that the energy stored in the elastic links suddenly releases when the links are broken. This phenomenon explains the superb resistance of the waiting structure: The energy of the projectile is spent to excite the waves of damage which are spread upon a large area and dissipate.

\subsection{NUMERICAL ALGORITHM}

Solving the system (1.11) numerically, we rewrite it as an autonomous system of first order differential equations:

$$
\begin{aligned}
\dot{\mathbf{z}_{\mathbf{i}}} & =\mathbf{p}_{\mathbf{i}}, \\
\dot{\mathbf{p}_{\mathbf{i}}} & =\frac{1}{m_{i}}\left(\phi_{i}-\gamma \mathbf{p}_{\mathbf{i}}\right),
\end{aligned}
$$

where

$$
\phi_{i}=\sum_{j \in N(i)} \frac{F_{i j}\left(\left|\mathbf{z}_{\mathbf{i}}-\mathbf{z}_{\mathbf{j}}\right|\right)}{\left|\mathbf{z}_{\mathbf{i}}-\mathbf{z}_{\mathbf{j}}\right|}\left(\mathbf{z}_{\mathbf{i}}-\mathbf{z}_{\mathbf{j}}\right) .
$$

Introducing the notation

$$
\overrightarrow{\mathbf{x}}=\left\{\mathbf{z}_{\mathbf{i}}, \mathbf{p}_{\mathbf{i}}\right\}, \quad \overrightarrow{\mathbf{f}}=\left\{\mathbf{p}_{\mathbf{i}}, \frac{1}{m_{i}}\left(\phi_{i}-\gamma \mathbf{p}_{\mathbf{i}}\right)\right\},
$$

we get

$$
\dot{\overrightarrow{\mathbf{x}}}=\overrightarrow{\mathbf{f}}(\overrightarrow{\mathbf{x}})
$$


We solve the resulting system via the second order Runge-Kutta method

$$
\overrightarrow{\mathbf{x}}_{n+1}=\overrightarrow{\mathbf{x}}_{n}+\frac{k}{2}\left(\overrightarrow{\mathbf{f}}\left(\overrightarrow{\mathbf{x}}_{n}\right)+\overrightarrow{\mathbf{f}}\left(\overrightarrow{\mathbf{x}}_{n}+h \overrightarrow{\mathbf{f}}\left(\overrightarrow{\mathbf{x}}_{n}\right)\right)\right),
$$

where $k$ denotes the time step. The stability condition of the numerical method depends on the speed $v_{d}$ from $(1.7),(1.9)$ of damage propagation and on the dissipation coefficient $\gamma$. In all numerical experiments that follow we establish convergence empirically via time step refinement.

\section{STRUCTURES UNDER A CONCENTRATED IMPACT}

In this section, we describe the resistance and failure of structures from waiting links impacted by a massive concentrated projectile. The kinetic energy of the projectile must be absorbed in the structure without its total failure.

\subsection{MODEL OF THE PROJECTILE}

The model takes into account penetration of the projectile through the structure and does not allow "slipping through" the line on knots. One cannot simply model the projectile as another "heavy knot" in the structure with an initial kinetic energy: In such a model the projectile would not be connected with the knots but the immediate neighbors; the projectile would slip through the net after the failure of the neighboring links.

In our simulation, the projectile is modeled as an "elastic ball" of the mass $M_{p}$ centered at the position $z_{p}$. Motion of the mass satisfies the equation

$$
M_{p} \ddot{\mathbf{z}_{\mathbf{p}}}=\sum_{j} \frac{F_{p j}\left(\left|\mathbf{z}_{\mathbf{p}}-\mathbf{z}_{\mathbf{j}}\right|\right)}{\left|\mathbf{z}_{\mathbf{p}}-\mathbf{z}_{\mathbf{j}}\right|}\left(\mathbf{z}_{\mathbf{p}}-\mathbf{z}_{\mathbf{j}}\right)
$$

where $j$ is the number of the knot in the structure. Equation 1.16 is similar to (1.11), but the force $F_{p j}$ is found from a different equation

$$
F_{p j}(z)= \begin{cases}0 & \text { if } z>B \\ \ln \left(\frac{B-A}{z-A}\right) & \text { if } \quad A<z \leq B \\ +\infty & \text { if } z \leq A\end{cases}
$$

In the numerical experiments that follow $A=0.5 L, B=2 L$.

This model states that the repulsive force $F_{p j}(z)$ is applied to the knots when the distance between them and the center $\mathbf{z}_{\mathbf{p}}$ of the projectile is smaller than a threshold $B$. This force grows when the distance decreases and becomes infinite when the distance is smaller than $A$. This model 
describes the projectile as a nonlinear elastic ball with a rigid nucleus. When it slips through the structure, the knots are repulsed from its path causing strain and/or breaking of the links.

\subsection{EFFECTIVENESS OF A DESIGN}

Comparing the history of damage of several designs, we need to work out a quantitative criterion of the effectiveness of the structure. This task is nontrivial, since different designs are differently damaged after the collision.

Effectiveness criterion. We use an integral criterion that is not sensitive to the details of the damage; All what we are measuring is the variation of the impulse of the projectile. It is assumed here that the projectile hits the structure falling down into it. To evaluate the effectiveness of the protective structure, we compute the scaled impulse $d$ - the ratio in the vertical component $p_{v}=\mathbf{p} \cdot(0,1)$ of the impulse $\mathbf{p}$ of the projectile before and after the impact:

$$
d=\frac{p_{v}\left(T_{\text {final }}\right)}{\left|p_{v}\left(T_{0}\right)\right|}
$$

where $T_{0}$ and $T_{\text {final }}$ are the initial and final moments of the observation, respectively. Notice that scaled impulse $d$ does not vary when the projectile is not in a contact with the structure; in this sense it is insensitive to the exact value of $T_{\text {final }}$.

Scaled impulse $d$ shows what portion of the impulse is passed to the structure from the projectile. It evaluates the structure's performance using the projectile as the measuring device. An elastic impact with a rigid body corresponds to the final impulse being opposite to the initial one $(d=-1)$. The absence of the structure correspond to $d=1$, because the impulse of the projectile does not change. If the projectile stops then $d=0$, if it breaks through the structure then $0<d \leq 1$, and if it is rejected then $-1 \leq d<0$. The smaller $d$ is, the more effective the structure is.

Other criteria. Other criteria compare the state of the structure before and after the collision. These criteria are applicable only if the structure (or its pieces) after the collision reaches a steady state. We register (i) the percentage of partially damaged links. (ii) the percentage of destroyed links. The first number shows how much damage spreads, and the second shows what percent of the structure is destroyed. Ideally, we wish to have a structure in which all elements are partially damaged, but no element is completely destroyed. 


\begin{tabular}{||l|l|l|l|l}
\hline Figure & $\alpha$ & $\begin{array}{l}\% \text { of damaged } \\
\text { links }\end{array}$ & $\begin{array}{l}\% \text { of destroyed } \\
\text { links }\end{array}$ & $\begin{array}{l}\text { Scaled final } \\
\text { impulse d }\end{array}$ \\
\hline \hline Figure 3(a) & 0.10 & $94 \%$ & $3.8 \%$ & -0.26 \\
\hline | Figure 3(b) & 0.25 & $42 \%$ & $3.8 \%$ & -0.32 \\
\hline$\|$ Figure 3(c) & 0.50 & $4.6 \%$ & $6.3 \%$ & 0.54
\end{tabular}

Table 1 Damage and/or destruction of a bridge.

Remark 4 The percentage of destroyed links is a rough criterion: It ignores the positions of the destroyed links.

\subsection{BRIDGE-LIKE DESIGNS}

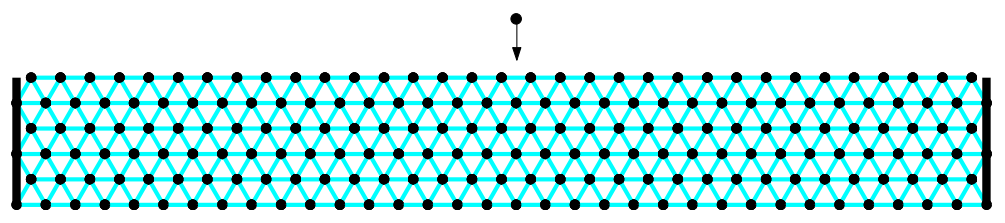

Figure 2 The bridge is supported from the sides. It is impacted by a projectile moving downward.

Finally, we discuss the simulation of damage of a bridge-like truss structure made from waiting links. We assume that the vertical sides of the structure are supported and the horizontal sides are free. A projectile that is modeled as "elastic ball" (3.1) impacts the center of the upper side of the structure moving vertically down with an initial speed $v_{0}$ (Figure 2).

The difference in the impulse of the projectile before and after impact shows the effectiveness of the structure. The number of destroyed links also represents the effectiveness showing how much the damage is spread. If the speed is small, the projectile is rejected; otherwise it penetrates through the structure.

We simulate the damage process of the bridge by varying the parameter $\alpha$ (the fraction of material put into the shorter link) while keeping the other parameters $\left(L, \Delta, z_{f_{1}}, z_{f_{2}}\right.$, total amount of material, etc.) the same for all runs. The results are summarized in Table 1 and Figure 3. 


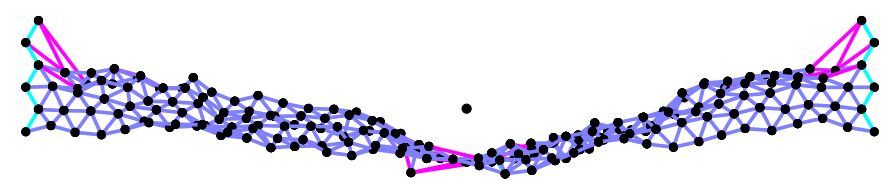

(a) $\alpha=0.10, T_{\text {final }}=500$, projectile is rejected.

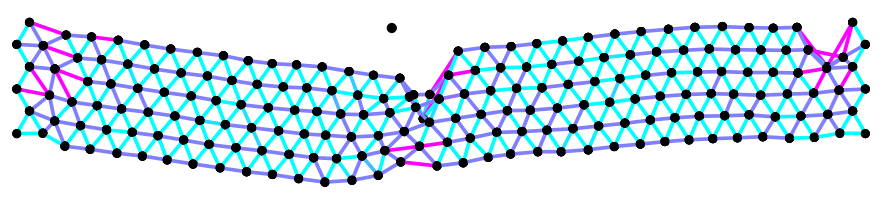

(b) $\alpha=0.25, T_{\text {final }}=500$, projectile is rejected.

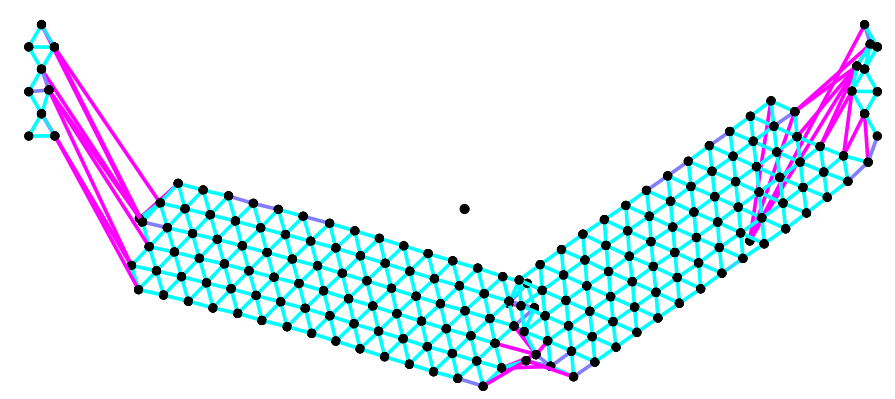

(c) $\alpha=0.50, T_{\text {final }}=250$, projectile goes through.

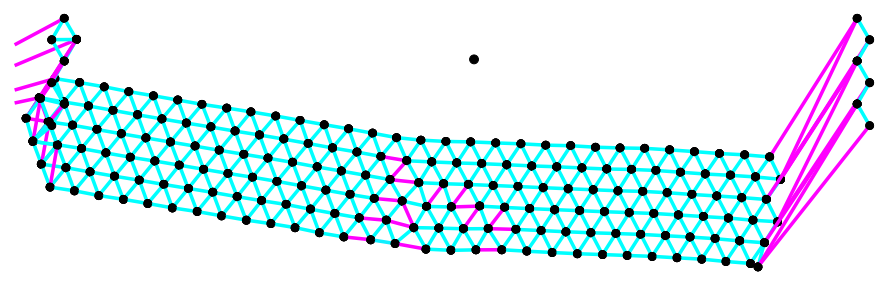

(d) $\alpha=1.00, T_{\text {final }}=250$, projectile goes through.

Figure 3 The final stages of the impacted bridges. The designs use the same amount of the materials, but differ in the parameter $\alpha$ : percentage of material used for the first (shorter) rod in the waiting link. 
One can see from Table 1 that as $\alpha$ decreases from 1.00 (conventional structure) to 0.10 the percentage of partially damaged links increases as the percentage of destroyed links decreases making the structure more resistant. Table 1 also shows that $\alpha=0.25$ is optimal for both minimizing the number of destroyed links and minimizing the scaled final impulse $d$ (see the discussion in Section 3.2).

Figure 3 shows the final stages of the impacted bridges. Intact waiting elements (both links are undamaged) are aquamarine; partially damaged elements (the short link is destroyed, the longer one is undamaged) are blue; destroyed elements (both links are damaged) are purple. The structures with $\alpha=.50$ and $\alpha=1.00$ (conventional structure) soon develop cracks and fall apart allowing the projectile to go through (see Figures $3(\mathrm{c}), 3(\mathrm{~d})$ ) while the structures with $\alpha=0.10$ and $\alpha=0.25$ preserve the structural integrity by dissipating energy and taking the stress away from the point of impact; this results in the rejection of the projectile (see Figures $3(\mathrm{a}), 3(\mathrm{~b})$ ). Notice that the final time $T_{\text {final }}$ is twice as small in the last two examples.

The propagation of the damage in the bridge is due to several factors: (i) local instabilities of the part of the network that contains a damaged link; (ii) the increase of force on neighboring links which allows the damage to spread; (iii) the waves that propagate through the network and initiate damage in areas remote from the point of collision.

\section{Acknowledgments}

The authors gratefully acknowledge support from ARO and from NSF.

\section{References}

[1] A. Cherkaev, and L. Slepyan. Waiting Element Structures and Stability under Extension. Int. J. Damage Mechanics, 1955, 4, No 1, 58-82.

[2] G. A. Francfort and J.-J. Marigo, Stable damage evolution in a brittle continuous medium. European J. Mech. A Solids 12 (1993), no. 2, 149-189

[3] Timoshenko, S.P; Goodier, J. N. Theory of Elasticity. 2d ed. McGraw-Hill Book Company, Inc., New York, Toronto, London, 1951.

[4] L.I. Slepyan, L.I. Mechanics of Cracks, Sudostroenie, Leningrad, 1990 (in Russian)

[5] Balk, A., Cherkaev, A. and Slepyan, L. Dynamics of Chains with Non-monotone Stress-strain Relations. 
I. Model and numerical experiments. J. Mech. Phys. Solids, 49, 2001, 131-148.

[6] Balk, A., Cherkaev, A. and Slepyan, L. Dynamics of Chains with Non-monotone Stress-strain Relations. II. Nonlinear Waves and Wave of Phase Transition. J. Mech. Phys. Solids, 49, 2001, 149-171. 